\title{
Perfect equilibrium in games with compact action spaces.
}

Citation for published version (APA):

Bajoori, E., Flesch, J., \& Vermeulen, A. J. (2011). Perfect equilibrium in games with compact action spaces. METEOR, Maastricht University School of Business and Economics. METEOR Research Memorandum No. 029 https://doi.org/10.26481/umamet.2011029

Document status and date:

Published: 01/01/2011

DOI:

10.26481/umamet.2011029

Document Version:

Publisher's PDF, also known as Version of record

\section{Please check the document version of this publication:}

- A submitted manuscript is the version of the article upon submission and before peer-review. There can be important differences between the submitted version and the official published version of record.

People interested in the research are advised to contact the author for the final version of the publication, or visit the DOI to the publisher's website.

- The final author version and the galley proof are versions of the publication after peer review.

- The final published version features the final layout of the paper including the volume, issue and page numbers.

Link to publication

\footnotetext{
General rights rights.

- You may freely distribute the URL identifying the publication in the public portal. please follow below link for the End User Agreement:

www.umlib.nl/taverne-license

Take down policy

If you believe that this document breaches copyright please contact us at:

repository@maastrichtuniversity.nl

providing details and we will investigate your claim.
}

Copyright and moral rights for the publications made accessible in the public portal are retained by the authors and/or other copyright owners and it is a condition of accessing publications that users recognise and abide by the legal requirements associated with these

- Users may download and print one copy of any publication from the public portal for the purpose of private study or research.

- You may not further distribute the material or use it for any profit-making activity or commercial gain

If the publication is distributed under the terms of Article $25 \mathrm{fa}$ of the Dutch Copyright Act, indicated by the "Taverne" license above, 


\section{Maastricht University}

Elnaz Bajoori, János Flesch,

Dries Vermeulen

Perfect equilibrium in games with compact action spaces

$\mathrm{RM} / 11 / 029$

\section{METEOR}

Maastricht University School of Business and Economics

Maastricht Research School of Economics

of Technology and Organization

P.O. Box 616

NL - 6200 MD Maastricht

The Netherlands 


\title{
Perfect equilibrium in games with compact action spaces
}

\author{
Elnaz Bajoori * JÁnos Flesch ${ }^{\dagger}$ \\ DRIES VERMEULEN $\ddagger$
}

April 26, 2011

\begin{abstract}
We investigate the relations between different types of perfect equilibrium, introduced by Simon and Stinchcombe (1995) for games with compact action spaces and continuous payoffs. Simon and Stinchcombe distinguish two approaches to perfect equilibrium in this context, the classical "trembling hand" approach, and the so-called "finitistic" approach. We propose an improved definition of the finitistic approach, called global-limit-offinite perfection, and prove its existence.

Despite the fact that the finitistic approach appeals to basic intuition, our results - specifically examples (1) and (2) - seem to imply a severe critique on this approach. In the first example any version of finitistic perfect equilibrium admits a Nash equilibrium strategy profile that is not limit admissible. The second example gives a completely mixed (and hence trembling hand perfect) Nash equilibrium that is not finitistically perfect.

Further examples illustrate the relations between the two approaches to perfect equilibrium and the relation to admissibility and undominatedness of strategies.
\end{abstract}

JEL Codes. C72.

Keywords. Strategic form games, perfect equilibrium, compact action spaces

\footnotetext{
e.bajoori@maastrichtuniversity.nl. Maastricht University, School of Business and Economics, Dept. of Quantitative Economics, Maastricht, The Netherlands.

${ }^{\dagger} j$.flesch@maastrichtuniversity.nl Maastricht University, School of Business and Economics, Dept. of Quantitative Economics, Maastricht, The Netherlands.

${ }^{\ddagger}$ Corresponding author, d.vermeulen@maastrichtuniversity.nl. Maastricht University, School of Business and Economics, Dept. of Quantitative Economics, Maastricht, The Netherlands., P.O. Box 616, 6200 MD Maastricht, The Netherlands.
} 


\section{Introduction}

In 1995 Simon and Stinchcombe [24], for brevity referred to as S\&S in this paper, defined perfect equilibrium for games with an infinite compact set of actions. They distinguished two main lines of definitions of perfection. The first line is based on the notion of a completely mixed strategy, giving rise to the notions of strongly perfect and weakly perfect equilibrium. This approach can be viewed as a direct generalization of the original trembling hand definition of perfect equilibrium introduced by Selten [23]. The second line of definitions, in S\&S referred to as the finitistic approach, uses the notion of an $\varepsilon$-perfect equilibrium in finite approximations of the original game. The resulting notion of perfect equilibrium is called limit-of-finite perfect equilibrium ${ }^{1}$. They showed that the first type of perfect equilibrium only admits limit admissible strategies. Furthermore, they conjectured that the two approaches are incomparable.

Perfect equilibrium in games with infinite action spaces is a useful tool to select Nash equilibria, for example in auction models with incomplete information, such as the ones studied in Compte and Jehiel [6] and Crémer et al. [7], where dominant strategies are not available as a selection criterion. Concrete applications of perfect equilibrium in infinite strategic form games can among others be found in Jackson et al. [11], S \& S [24], and Anderson et al. [2].

In this paper we investigate the existing relations between the various types of perfect equilibrium within the framework of strategic form games with compact action spaces and continuous payoff functions.

The diagram below gives an overview of those relations between different types of perfect equilibrium that are currently known. Strongly perfect, weakly perfect, and lof (limit-of-finite) perfect equilibrium are introduced by S\&S. Strongly glof (global limit-of-finite) and weakly glof perfect equilibrium are introduced in this paper. Limit undominated Nash equilibrium, a stronger version of limit admissibility from S\&S, is also introduced in this paper.

\footnotetext{
${ }^{1}$ Simon and Stinchcombe also introduce another variation on this type of perfection, called anchored perfection. In this paper we hardly ever consider this variation though.
} 


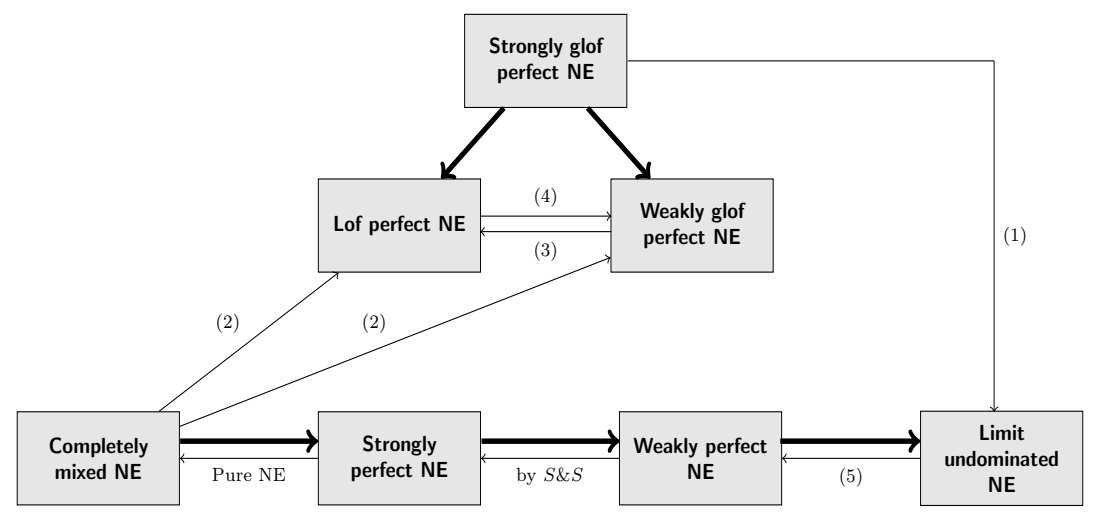

Boldface arrows indicate valid implications. For example, every strongly glof perfect equilibrium is a lof perfect equilibrium. Roman face arrows indicate implications that do not hold in general. Counterexamples are provided by previous studies, and examples (1) to (5) in this paper.

Related literature Equilibrium selection and refinement theory already have a long and steady-going tradition, arguably starting with essential equilibrium (Wu Wen-Tsün and Jiang Jia-He [26]), perfect equilibrium (Selten [23]) and proper equilibrium (Myerson [20]) for finite strategic form games. Theory on refinements for games with compact action spaces has been developed in, among others, Méndez et al. [15], Simon and Stinchcombe [24], and CarbonellNicolau [4]. Developments on refinements in the context of extensive form games are for example sequential equilibrium (Kreps and Wilson [14]) and theory on strategic stability (Kohlberg and Mertens [13], Hillas [10], Mertens [16] [17], and more recently Govindan and Wilson [9]).

The above line of literature mainly concerns what Andersson et al. [2] refer to as "strategic uncertainty" where a player is required to play strategies that guarantee robustness against possible mistakes by a player's opponents. An alternative line of research on equilibrium refinement is on "structural uncertainty" where, due to informational uncertainty, iterated deletion of dominated strategies is used as a refinement technique. This approach, also known as the global games approach, was developed by Carlsson and van Damme [5], with applications in for example banking and finance (Morris and Shin [19]). 
Results The paper makes two main contributions. The first contribution is the introduction of global limit-of-finite perfection, from now on referred to as glof perfection. S\&S already introduced lof perfect equilibrium as the limit of $\varepsilon$-perfect equilibria of large but finite approximations of the original game. Despite its relative straightforward intuition, lof perfect equilibrium does not always select available dominant strategies.

Strongly glof perfect equilibrium is defined in the same way as lof perfect equilibrium, but with the added requirement that best responses in the approximating games are in fact best responses in the original game. Strongly glof perfect equilibrium is a stronger version of finitistic perfection than lof perfect equilibrium. Weakly glof perfect equilibrium adds to the definition of lof perfection the weak requirement that best responses in the approximating games are only close to best responses in the original game. We show existence of weakly glof perfect equilibrium. Moreover, we show that weakly (and therefore also strongly) glof perfect equilibrium does uniquely select dominant strategy Nash equilibria as soon as dominant strategies are available to a player.

Second, we provide five illustrative counterexamples. Example 1 presents a strongly glof perfect equilibrium that is not limit admissible. This result is in line with Example 2.4 of $S \& S$ of a lof perfect equilibrium that is not limit admissible. Example 2 verifies the conjecture of Simon and Stinchcombe that strong perfection does not imply finitistic perfection. More precisely, it is an example a completely mixed, and therefore strongly perfect, equilibrium that is neither lof pefect (and therefore also not anchored perfect) nor weakly (and therefore also not strongly) glof perfect. Example 3 presents a weakly glof perfect equilibrium that is not lof perfect, and therefore also not strongly glof perfect. Example 4 is in fact equivalent to Example 2.4 of S\&S with the added feature that the action spaces are intervals instead of the union of an interval and an isolated point.

Example 5 is a comment on the theorem of van Damme that for bimatrix games a Nash equilibrium is perfect precisely when the equilibrium strategies are undominated. This equivalence no longer holds in the more general context of two-player games with compact action spaces and continuous payoff functions. 
Example 5 presents a Nash equilibrium of a $2 \times(\infty+1)$-bimatrix game in undominated strategies that is not weakly perfect. An added feature of the example is that there is another Nash equilibrium in this game that is payoff equivalent to the first Nash equilibrium, while the second Nash equilibrium is weakly perfect. Thus, weakly perfect equilibrium is not invariant, in contrast to perfect equilibrium for finite strategic form games. The example highlights the fact that for infinite compact action spaces, the topology on the action spaces starts to matter for perfection.

Except for Example 4, all our examples use countably infinite pure action spaces. We specifically chose not to use compact intervals as action spaces, in order to highlight the underlying logic of the constructions. It is nevertheless evident from the constructions that, with a bit (well, for some examples perhaps a bit more than a bit) of extra work the examples can also be transformed to counterexamples on the unit interval.

\section{Preliminaries}

For a metric space $(X, d)$, a set $U \subseteq X$ is open if for every $x \in U$ there is an $\varepsilon>0$ such that if $y \in X$ and $d(x, y)<\varepsilon$ then $y \in U$. The topology on $X$ induced by metric $d$ is the collection of all open sets we can thus construct. For a topological space $X$, the Borel $\sigma$-field on $X$ is the smallest $\sigma$-field that contains all open sets. A probability measure on $X$ is a function $\mu$ from a $\sigma$-field $\Sigma$ on $X$ to $[0,1]$ such that $\mu(X)=1$, and moreover

$$
\mu\left(\bigcup_{i \in I} E_{i}\right)=\sum_{i \in I} \mu\left(E_{i}\right)
$$

for every countable collection $\left(E_{i}\right)_{i \in I}$ of pairwise disjoint sets in $\Sigma$. For a compact set $X, \Delta(X)$ denotes the set of probability measures on the Borel $\sigma$-field on $X$. A probability measure $\mu \in \Delta(X)$ is completely mixed if $\mu(U)>0$ for every non-empty open subset $U$ of $X$.

In this paper we use two different metrics on the space $\Delta(X)$. For a non-empty set $B \subseteq X$, the distance $d(x, B)$ between a point $x \in X$ and the set $B$ is

$$
d(x, B)=\inf \{d(x, y) \mid y \in B\}
$$


The $\varepsilon$-neighborhood of $B$ is $B^{\varepsilon}=\{x \in X \mid d(x, B)<\varepsilon\}$. The weak metric is defined for $\mu, \nu \in \Delta(X)$ by

$$
\rho^{w}(\mu, \nu)=\inf \left\{\varepsilon \mid \forall B \in \Sigma: \mu(B) \leq \nu\left(B^{\varepsilon}\right)+\varepsilon \text { and } \nu(B) \leq \mu\left(B^{\varepsilon}\right)+\varepsilon\right\}
$$

and the strong metric by

$$
\rho^{s}(\mu, \nu)=\sup \{|\mu(B)-\nu(B)| \mid B \in \Sigma\} .
$$

Note that for every $\mu$ and $\nu, \rho^{w}(\mu, \nu) \leq \rho^{s}(\mu, \nu)$. This easily follows from the observation that for every $B \in \Sigma$, both $\mu(B)-\nu\left(B^{\varepsilon}\right)$ and $\nu(B)-\mu\left(B^{\varepsilon}\right)$ are smaller than or equal to $|\mu(B)-\nu(B)|$ and therefore smaller than or equal to $\rho^{s}(\mu, \nu)$ as well.

The weak (strong) topology on $\Delta(X)$ is the topology induced by the weak (strong) metric. The inequality $\rho^{w}(\mu, \nu) \leq \rho^{s}(\mu, \nu)$ implies that the weak topology is included in the strong topology. Since $X$ is compact, $\Delta(X)$ is compact with respect to the weak metric. Further, compactness and sequential compactness are equivalent for metric spaces.

\section{Strategic form games}

In this section we introduce the game theoretic notation used in this paper. A strategic form game (with compact action spaces) is a triplet $\Gamma=(N, A, u)$ where $N=\{1, \ldots, n\}$ is the set of the players, $A=\prod_{i \in N} A_{i}$ is the set of profiles of actions, and $u=\left(u_{1}, \ldots, u_{n}\right)$ is the vector of payoff functions. We assume for every player $i \in N$ that the set $A_{i}$ of actions is a non-empty compact subset of $\mathbb{R}$, and that the payoff functions $u_{i}: A \rightarrow \mathbb{R}$ are continuous ${ }^{2}$.

The set $\Delta\left(A_{i}\right)$ is the set of (mixed) strategies of player $i$. We identify every action $a_{i}$ with the Dirac measure $\delta\left(a_{i}\right)$, the strategy that selects $a_{i}$ with probability 1. A vector $\sigma=\left(\sigma_{i}\right)_{i \in N}$ with $\sigma_{i} \in \Delta\left(A_{i}\right)$ for all $i$ is called a strategy profile. The set of strategy profiles is denoted ${ }^{3}$ by $\Delta(A)$. For every strategy

\footnotetext{
${ }^{2}$ Everything we do in this paper immediately generalizes to general compact metric spaces. In this context note that a compact metric space is automatically separable, so that the finitistic approach also has a bite in the general case.

${ }^{3}$ With a slight abuse of notation we implicitly identify each strategy profile $\sigma=\left(\sigma_{i}\right)_{i \in N}$ with its induced probability distribution in $\Delta(A)$. Obviously not every element of $\Delta(A)$ can be obtained this way. We restrict attention to the independent elements of $\Delta(A)$ that are induced by strategy profiles.
} 
profile $\sigma \in \Delta(A)$ we define $u_{i}(\sigma)=\int u_{i} d \sigma$. Prokhorov's Theorem implies that $u_{i}(\sigma)$ is continuous in $\sigma$ with respect to the weak metric $\rho^{w}$ on $\Delta(A)^{4}$.

We write $A_{-i}=\prod_{j \in N \backslash\{i\}} A_{j}$. A generic element of $\Delta\left(A_{-i}\right)$ is denoted by $\sigma_{-i}=$ $\left(\sigma_{j}\right)_{j \neq i}$. By $\left(\sigma_{-i}, \tau_{i}\right)$ or $\left(\sigma \mid \tau_{i}\right)$ we denote the strategy profile where every player $j \neq i$ adheres to strategy $\sigma_{j}$, while player $i$ uses $\tau_{i}$. A strategy $\tau_{i} \in \Delta\left(A_{i}\right)$ is called a best response of player $i$ to the strategy profile $\sigma \in \Delta(A)$ if $u_{i}(\sigma \mid$ $\left.\tau_{i}\right) \geq u_{i}\left(\sigma \mid \rho_{i}\right)$ for all $\rho_{i} \in \Delta\left(A_{i}\right)$. A strategy profile $\sigma=\left(\sigma_{i}\right)_{i \in N}$ is a Nash equilibrium if

$$
u_{i}(\sigma) \geq u_{i}\left(\sigma \mid \tau_{i}\right)
$$

for all $i$ and all $\tau_{i} \in \Delta\left(A_{i}\right)$. So, $\sigma$ is a Nash equilibrium if each $\sigma_{i}$ is a best response to $\sigma$.

For a strategy profile $\sigma, B R_{i}(\sigma)$ is the set of best responses of player $i$ to $\sigma$. An action $a_{i} \in A_{i}$ is a pure best response of player $i$ to $\sigma$ if $\delta\left(a_{i}\right) \in B R_{i}(\sigma)$. By $\operatorname{PBR}_{i}(\sigma)$ we denote the set of pure best responses of player $i$ to $\sigma$. The set $\mathrm{PBR}_{i}(\sigma)$ is non-empty by compactness of $A_{i}$ and continuity of $u_{i}$. Note that $\sigma_{i}$ is a best response to $\sigma$ if and only if it puts weight 1 on pure best responses, i.e., $\sigma_{i}\left(P B R_{i}(\sigma)\right)=1$. For a non-empty compact subset $X_{i}$ of $A_{i}$,

$$
B R_{i}\left(\sigma \mid X_{i}\right)=\left\{\rho_{i} \in \Delta\left(X_{i}\right) \mid u_{i}\left(\sigma \mid \rho_{i}\right) \geq u_{i}\left(\sigma \mid \tau_{i}\right) \text { for all } \tau_{i} \in \Delta\left(X_{i}\right)\right\}
$$

is the set of player $i$ 's best strategies against $\sigma$ given that the pure choices of player $i$ are restricted to actions in $X_{i}$. By $P B R_{i}\left(\sigma \mid X_{i}\right)$ we denote the set of those actions of player $i$ that belong to $B R_{i}\left(\sigma \mid X_{i}\right)$.

\section{Perfect equilibrium}

Selten [23] introduced the notion of perfect equilibrium for non-cooperative extensive form games. The definition of perfect equilibrium is based on the idea that a satisfactory interpretation of equilibrium points should accommodate possible slight mistakes. Selten showed that perfect equilibrium exists for finite extensive form games with perfect recall.

\footnotetext{
${ }^{4}$ Throughout the paper we use several results from real analysis without further mention. All of the results we use are well-known. Good references for the relevant theory are for example Aliprantis and Border [1], and Billingsley [3].
} 
Twenty years later Simon and Stinchcombe [24] provided a general treatment of perfect equilibria for infinite strategic form games with compact action spaces and continuous payoffs. S\&S discuss three distinct approaches to perfect equilibrium for infinite normal form games. In the first two approaches, as in Selten's original definition, players may "tremble", modeled by having players choose completely mixed strategies. Players are then required to play approximate best responses to opponents' trembling strategies. In the strong approach, a tremble assigns high probability to the set of pure best responses itself, while in the weak approach a tremble is only required to assign high probability to a neighborhood of this set. The third, limit-of-finite, approach applies traditional refinements to sequences of successively larger finite approximations of the original infinite game. S\&S proved the existence of these three generalized notions of perfect equilibrium. Moreover, they showed that the set of strongly perfect equilibria is a closed, non-empty subset of the set of weakly perfect equilibria, which is a closed subset of the collection of limit admissible Nash equilibria.

Both the strong and weak approaches of S\&S use a direct generalization of the notion of completely mixed strategies in the definition of perfect equilibrium. Let $\epsilon>0$. A completely mixed strategy profile $\sigma=\left(\sigma_{i}\right)_{i \in N}$ in $\Delta(A)$ is a strongly $\varepsilon$-perfect equilibrium if for every player $i \in N$ it holds that $\rho^{s}\left(\sigma_{i}, B R_{i}(\sigma)\right)<\epsilon$. It is a weakly $\epsilon$-perfect equilibrium if $\rho^{w}\left(\sigma_{i}, B R_{i}(\sigma)\right)<\epsilon$.

Definition 4.1 (Simon and Stinchcombe) A strategy profile $\sigma$ is a strongly (respectively weakly) perfect equilibrium if there is a sequence $\left(\sigma^{k}\right)_{k=1}^{\infty}$ of strongly (weakly) $\frac{1}{k}$-perfect equilibria with $\rho^{w}\left(\sigma^{k}, \sigma\right) \rightarrow 0$ as $k \rightarrow \infty$.

In order to guarantee existence of limit points, the above definition only considers weak limits of $\varepsilon$-perfect equilibria. Finitistic perfection is based on a different approach where games with infinite action spaces are approximated by games with large, but still finite, action spaces.

Definition 4.2 (Simon and Stinchcombe) A strategy profile $\sigma=\left(\sigma_{i}\right)_{i \in N}$ is a limit-of-finite (lof) perfect equilibrium if for every player $i \in N$ there is a sequence $\left(B_{i}^{k}\right)_{k=1}^{\infty}$ of finite subsets of $A_{i}$ and a sequence $\left(\sigma_{i}^{k}\right)_{k=1}^{\infty}$ of completely mixed strategies in $\Delta\left(B_{i}^{k}\right)$ such that for every $i \in N$ 
[1] for every $\varepsilon>0$ there is a $K \in \mathbb{N}$ such that for each $k>K$ and for each $a_{i} \in A_{i}$ there is a $b_{i} \in B_{i}^{k}$ with $\left|a_{i}-b_{i}\right|<\varepsilon$

$[2] \rho^{w}\left(\sigma_{i}^{k}, \sigma_{i}\right) \rightarrow 0$ as $k \rightarrow \infty$

[3] $\sum_{x \in P B R_{i}\left(\sigma^{k} \mid B_{i}^{k}\right)} \sigma_{i}^{k}(x) \rightarrow 1$ as $k \rightarrow \infty \quad\left(\right.$ where $\left.\sigma^{k}=\left(\sigma_{i}^{k}\right)_{i \in N}\right)$.

This definition has quite some appeal given our basic intuition that infinite games often serve as a proxy for large finite games, and that therefore strategic behavior should carry over from the finite setting to infinite games. Despite this basic intuition, S\&S already noted that even in games with dominant actions lof perfection may fail to eliminate Nash equilibria that do not select this dominant action. In order to circumvent this drawback, we propose the following modification of lof perfection, which exclusively selects the dominant strategies in case such strategies are available to a player.

Definition 4.3 A strategy profile $\sigma=\left(\sigma_{i}\right)_{i \in N}$ is a strongly global-limitof-finite (strongly glof) perfect equilibrium if in Definition 4.2 condition 3) is replaced by

[4] $\rho^{s}\left(\sigma_{i}^{k}, B R_{i}\left(\sigma^{k}\right)\right) \rightarrow 0$ as $k \rightarrow \infty$.

The strategy profile $\sigma=\left(\sigma_{i}\right)_{i \in N}$ is a weakly glof perfect equilibrium if in Definition 4.2 condition 3 ) is replaced by

[5] $\rho^{w}\left(\sigma_{i}^{k}, B R_{i}\left(\sigma^{k}\right)\right) \rightarrow 0$ as $k \rightarrow \infty$.

S\&S introduced the method of anchoring as a-partial-remedy to the failure of lof perfection to select limit admissible strategies. Anchoring requires us to select a priori a collection of actions that are considered to be indispensable for a player. Our approach is more flexible in the sense that the indispensability of selected strategies is allowed to be contingent on the approximating sequence of strategy profiles we consider.

Simon and Stinchcombe established existence of lof perfect equilibrium. We show that weakly glof perfect equilibrium exists, and that every weakly glof perfect equilibrium is a Nash equilibrium. The rather lengthy proof is deferred to Appendix A. 
Theorem 4.4 Every strategic form game with compact action spaces has a weakly glof perfect equilibrium. Moreover, every weakly glof perfect equilibrium is a Nash equilibrium.

Since clearly every strongly glof perfect equilibrium is also weakly glof, both strong and weak glof perfection refines the set of Nash equilibria. Existence of strongly glof perfect equilibrium remains an open question.

\section{Admissibility}

In this section we analyze the relation between the various notions of perfection and admissibility. We show that weak perfection exclusively selects limit undominated strategies. We also show that this statement is a genuine generalization of the classical result for finite games that perfection implies undominatedness. For the finitistic approach the connection to admissibility is much weaker. As said before, in Example 2.4 S\&S already present a lof perfect equilibrium that does not select the unique dominant strategy. We show that glof perfection performs better: for every game in which a player has dominant strategies, glof perfect equilibrium exclusively selects equilibria in which such a player plays one of his dominant strategies. However, the analogous result for undominated strategies again fails to hold. Example 1 presents a two-player game with a strongly glof perfect equilibrium in which both players play dominated strategies that are not limits of undominated strategies.

We say that $\tau_{i} \in \Delta\left(A_{i}\right)$ dominates $\sigma_{i} \in \Delta\left(A_{i}\right)$ if

$$
u_{i}\left(\sigma \mid \tau_{i}\right) \geq u_{i}\left(\sigma \mid \sigma_{i}\right)
$$

for all strategy profiles $\sigma \in \Delta(A)$, and the inequality is strict for at least one strategy profile $\sigma \in \Delta(A)$. A strategy $\tau_{i}$ is dominant if $u_{i}\left(\sigma \mid \tau_{i}\right) \geq u_{i}\left(\sigma \mid \sigma_{i}\right)$ for all $\sigma \in \Delta(A)$ and $\sigma_{i} \in \Delta\left(A_{i}\right)$. The set of all dominant strategies for player $i$ is denoted by $D_{i}$. Obviously $D_{i}$ may be empty.

If there is no $\tau_{i}$ that dominates $\sigma_{i}$, we say that $\sigma_{i}$ is undominated. We denote the set of undominated strategies of player $i$ by $U_{i}$. We write $\sigma_{i} \in L U_{i}$ if there exists a sequence $\left(\sigma_{i}^{k}\right)_{k \in \mathbb{N}}$ such that $\rho^{w}\left(\sigma_{i}^{k}, \sigma_{i}\right) \rightarrow 0$ as $k \rightarrow \infty$, and $\sigma_{i}^{k}$ is undominated 
for all $k^{5}$. For $a_{i} \in A_{i}$ we write $a_{i} \in P U_{i}$, if $a_{i}$ is not dominated by any $b_{i} \in A_{i}$. Following S\&S (1995), a strategy $\sigma_{i}$ is limit admissible if $\sigma_{i}\left(\overline{P U_{i}}\right)=1$, where $\overline{P U_{i}}$ denotes the closure of the set $P U_{i}$.

Every limit undominated strategy is also limit admissible. The converse does not hold though, not even for finite strategic form games. In the table below the payoff matrix of player 2 is given:

$$
u_{2}=\left[\begin{array}{lll}
4 & 0 & 3 \\
0 & 4 & 3
\end{array}\right]
$$

The strategy $\sigma_{2}=\left(\frac{1}{2}, \frac{1}{2}, 0\right)$ is limit admissible for player 2. Nevertheless, $\sigma_{2}$ is dominated by $(0,0,1)$.

\subsection{Weak perfection and admissibility}

S\&S already showed that weakly perfect equilibrium is limit admissible. In this section we show the somewhat stronger claim that weakly perfect equilibrium strategies are limit undominated. We also show that this is the exact analogue of the classical result for finite games that perfect equilibrium strategies are undominated.

Theorem 5.1 Let $\sigma$ be a weakly perfect equilibrium. Then $\sigma_{i} \in L U_{i}$ for each $i$.

Proof. By Definition 4.1 and Theorem 3.2 of Billingsley [3], there exists a sequence of completely mixed strategy profiles $\sigma^{k}=\left(\sigma_{i}^{k}\right)_{i \in N}$ such that for every $i \in N$ we have $\rho^{w}\left(\sigma_{i}^{k}, \sigma_{i}\right) \rightarrow 0$ and $\rho^{w}\left(\sigma_{i}^{k}, B R_{i}\left(\sigma^{k}\right)\right) \rightarrow 0$ as $k \rightarrow \infty$.

So, for every $i \in N$, there is a sequence $\tau_{i}^{k} \in B R_{i}\left(\sigma^{k}\right)$ with $\rho^{w}\left(\sigma_{i}^{k}, \tau_{i}^{k}\right) \rightarrow 0$ as $k \rightarrow \infty$. Therefore, by the triangle inequality, $\rho^{w}\left(\tau_{i}^{k}, \sigma_{i}\right) \rightarrow 0$ as $k \rightarrow \infty$. Since $\tau_{i}^{k}$ is a best response against the completely mixed profile $\sigma^{k}$, by Lemma B.2, $\tau_{i}^{k}$ is undominated. Therefore $\sigma_{i}$ is limit undominated.

At first glance Theorem 5.1 seems to be a compromise between the well-known result for finite normal form games that the strategies in a perfect equilibrium are undominated, and the transition to infinite compact action spaces. The following Proposition shows that this compromise is only seemingly, since for finite games limit undominated strategies are in fact undominated.

\footnotetext{
${ }^{5}$ In other words, the set $L U_{i}$ is the closure of the set $U_{i}$ under the weak metric. The letters $L U$ stand for limit undominated.
} 
Proposition 5.2 For strategic form games with finite action spaces, the set $U_{i}$ is closed, so that $U_{i}=L U_{i}$ for such games.

Proof. To prove that the set of undominated strategies is closed, we show that the set of dominated strategies is open. Suppose $\sigma_{i}$ is dominated by $\tau_{i}$. We prove that there is an open set $V \ni \sigma_{i}$ such that every $\nu_{i} \in V$ is dominated as well. Define $\varepsilon=\frac{1}{2} \min \left\{\sigma_{i}(a) \mid a \in A_{i}\right.$ and $\left.\sigma_{i}(a)>0\right\}$. Since $A_{i}$ is a finite set, $\varepsilon>0$. Define

$$
V=\left\{\nu_{i} \in \Delta\left(A_{i}\right) \mid\left\|\nu_{i}-\sigma_{i}\right\|_{\infty}<\varepsilon\right\}
$$

Here, $\|\cdot\|_{\infty}$ denotes the maximum norm. Take $\nu_{i} \in V$. Write $\mu_{i}=2 \nu_{i}-\sigma_{i}$. We show that $\mu_{i} \in \Delta\left(A_{i}\right)$. Note that if $\sigma_{i}(a)=0$ then $\mu_{i}(a) \geq 0$. Now assume that $\sigma_{i}(a)>0$. Since $\left\|\nu_{i}-\sigma_{i}\right\|_{\infty}<\varepsilon$, we have

$$
\left|\nu_{i}(a)-\sigma_{i}(a)\right|<\varepsilon \leq \frac{1}{2} \sigma_{i}(a)
$$

Consequently, $\mu_{i}(a)=2 \nu_{i}(a)-\sigma_{i}(a)=2 \nu_{i}(a)-2 \sigma_{i}(a)+\sigma_{i}(a)>0$. Because $\sum_{a \in A_{i}} \mu_{i}(a)=1$ clearly holds, we obtain $\mu_{i} \in \Delta\left(A_{i}\right)$.

We show that $\nu_{i}=\frac{1}{2} \sigma_{i}+\frac{1}{2} \mu_{i}$ is dominated by $\frac{1}{2} \tau_{i}+\frac{1}{2} \mu_{i}$. As $\sigma_{i}$ is dominated by $\tau_{i}$, there is a strategy profile $\rho_{-i} \in \Delta\left(A_{-i}\right)$ such that $u_{i}\left(\rho_{-i}, \tau_{i}\right)>u_{i}\left(\rho_{-i}, \sigma_{i}\right)$. Since $u_{i}$ is linear in each player's strategy we have

$$
\begin{aligned}
u_{i}\left(\rho_{-i}, \frac{1}{2} \tau_{i}+\frac{1}{2} \mu_{i}\right) & =\frac{1}{2} u_{i}\left(\rho_{-i}, \tau_{i}\right)+\frac{1}{2} u_{i}\left(\rho_{-i}, \mu_{i}\right) \\
& >\frac{1}{2} u_{i}\left(\rho_{-i}, \sigma_{i}\right)+\frac{1}{2} u_{i}\left(\rho_{-i}, \mu_{i}\right) \\
& =u_{i}\left(\rho_{-i}, \frac{1}{2} \sigma_{i}+\frac{1}{2} \mu_{i}\right) .
\end{aligned}
$$

Similarly we conclude for every $\sigma_{-i} \in \Delta\left(A_{-i}\right)$ that

$$
u_{i}\left(\sigma_{-i}, \frac{1}{2} \tau_{i}+\frac{1}{2} \mu_{i}\right) \geq u_{i}\left(\sigma_{-i}, \frac{1}{2} \sigma_{i}+\frac{1}{2} \mu_{i}\right)
$$

Hence, $\nu_{i}=\frac{1}{2} \sigma_{i}+\frac{1}{2} \mu_{i}$ is dominated by $\frac{1}{2} \tau_{i}+\frac{1}{2} \mu_{i}$.

\subsection{Finitistic perfection and admissibility}

The relation between finitistic perfection and admissibility is much weaker than it is for weakly perfect equilibrium. S\&S already have an example of a game in which one of the players has a unique dominant action (see example 2.4 of Simon 
and Stinchcombe [24]), while nevertheless there is a lof perfect equilibrium in this game in which the player in question does play a dominated action. Hence, even in games with dominant actions, lof perfection does not exclusively select limit admissible strategies.

We show that our alternative definition of finitistic perfection, glof perfect equilibrium, does select only dominant strategies in case such strategies are available to a player. In an example we show that for games without dominant strategies also glof perfect equilibrium may use strategies that are not limit admissible.

Write $P D_{i}:=\left\{a_{i} \in A_{i} \mid \delta\left(a_{i}\right) \in D_{i}\right\}$. In Appendix B we show that $D_{i}$ is closed with respect to the weak metric in $\Delta\left(A_{i}\right)$, that $P D_{i}$ is closed in $A_{i}$, and that $D_{i}=\Delta\left(P D_{i}\right)$.

Theorem 5.3 Let $\sigma \in \Delta(A)$ be a weakly glof equilibrium. Suppose that $D_{i}$ is not empty. Then $\sigma_{i} \in D_{i}$.

Proof. Because $\sigma$ is a weakly glof equilibrium, for every player $i$ there is a sequence $\left(\sigma_{i}^{k}\right)_{k=1}^{\infty}$ such that $\rho^{w}\left(\sigma_{i}^{k}, \sigma_{i}\right) \rightarrow 0$ and $\rho^{w}\left(\sigma_{i}^{k}, B R_{i}\left(\sigma^{k}\right)\right) \rightarrow 0$ as $k \rightarrow \infty$. So, for every $k$ we can take $\tau_{i}^{k} \in B R_{i}\left(\sigma^{k}\right)$ such that $\rho^{w}\left(\sigma_{i}^{k}, \tau_{i}^{k}\right) \rightarrow 0$ as $k \rightarrow \infty$. Then, by the triangle inequality for $\rho^{w}$, also $\rho^{w}\left(\sigma_{i}, \tau_{i}^{k}\right) \rightarrow 0$ as $k \rightarrow \infty$.

Suppose that $D_{i}$ is not empty. Then, by Proposition B.4, $P D_{i}$ is not empty either. Let $U \supset P D_{i}$ be open. First we show that $\tau_{i}^{k}(U)=1$ for sufficiently large $k$. When $U=A_{i}$, this is evident. Suppose $U \neq A_{i}$. Take $a_{i} \in A_{i} \backslash U$ and $b_{i} \in P D_{i}$. By Lemma B.1, there is an $a_{-i} \in A_{-i}$ such that

$$
u_{i}\left(b_{i}, a_{-i}\right)>u_{i}\left(a_{i}, a_{-i}\right) .
$$

Since $u_{i}$ is continuous, there are open sets $V\left(a_{i}\right) \ni a_{i}$ and $W\left(a_{-i}\right) \ni a_{-i}$ such that for every $x_{i} \in V\left(a_{i}\right)$ and $x_{-i} \in W\left(a_{-i}\right)$ we have

$$
u_{i}\left(b_{i}, x_{-i}\right)>u_{i}\left(x_{i}, x_{-i}\right) .
$$

Take $x_{i} \in V\left(a_{i}\right)$. Since $b_{i}$ is a dominant strategy, we have

$$
u_{i}\left(b_{i}, y_{-i}\right) \geq u_{i}\left(x_{i}, y_{-i}\right)
$$

for every $y_{-i} \in A_{-i}$. Further, define $B_{-i}^{k}=\prod_{j \neq i} B_{j}^{k}$. Then $B_{-i}^{k} \cap W\left(a_{-i}\right) \neq \emptyset$ for large $k$ by Definition 4.3. So, since $\sigma_{-i}^{k}$ is a completely mixed strategy in 
$\Delta\left(B_{-i}^{k}\right)$, we know that

$$
u_{i}\left(b_{i}, \sigma_{-i}^{k}\right)>u_{i}\left(x_{i}, \sigma_{-i}^{k}\right)
$$

for large $k$. Hence, for large $k, x_{i}$ is not a best response to $\sigma^{k}$. We conclude for every $a_{i} \in A_{i} \backslash U$ that

$$
V\left(a_{i}\right) \cap P B R_{i}\left(\sigma^{k}\right)=\emptyset
$$

for large $k$. Now note that $A_{i} \backslash U$ is compact, since it is a closed subset of a compact set. Therefore, there are actions $a_{i}^{1}, \ldots, a_{i}^{p}$ in $A_{i} \backslash U$ such that $A_{i} \backslash U$ is a subset of $\bigcup_{r=1}^{p} V\left(a_{i}^{r}\right)$. Moreover,

$$
\operatorname{PBR}_{i}\left(\sigma^{k}\right) \cap \bigcup_{j=1}^{p} V\left(a_{i}^{j}\right)=\emptyset
$$

for large $k$. Consequently $P B R_{i}\left(\sigma^{k}\right) \subseteq U$ for large $k$. But $\tau_{i}^{k} \in B R_{i}\left(\sigma^{k}\right)$, so that $\tau_{i}^{k}\left(P B R_{i}\left(\sigma^{k}\right)\right)=1$ for all $k$. Hence, $\tau_{i}^{k}(U)=1$ for large $k$, as claimed.

We show that $\sigma_{i} \in D_{i}$. Take $\varepsilon>0$. Since $\rho^{w}\left(\sigma_{i}, \tau_{i}^{k}\right) \rightarrow 0$ as $k \rightarrow \infty$,

$$
\tau_{i}^{k}(U)-\varepsilon \leq \sigma_{i}\left(U^{\varepsilon}\right)
$$

for large $k$. Because $\tau_{i}^{k}(U)=1$ for large $k$, we get $1-\varepsilon \leq \sigma_{i}\left(U^{\varepsilon}\right)$. Since $\varepsilon>0$ and $U \supseteq P D_{i}$ were chosen arbitrarily, and since $P D_{i}$ is a closed set, we can conclude that $\sigma_{i}\left(P D_{i}\right)=1$. Hence, $\sigma_{i} \in \Delta\left(P D_{i}\right)=D_{i}$.

Theorem 5.3 shows that in games where players have dominant strategies, weak glof perfection can only select those equilibria in which dominant strategies are played. However, the next example shows that, in games where players do not have dominant strategies, even strong glof perfection may fail to uniquely select limit admissible strategies.

EXAMPLE 1. The action spaces are

$$
A_{1}=A_{2}=\{-\infty, \ldots,-3,-2,-1,1,2,3, \ldots, \infty\},
$$

in which each integer is an isolated point, whereas $-\infty$ is the limit of the sequence $-1,-2, \ldots$ and $\infty$ is the limit of the sequence $1,2, \ldots$ The topological structure is shown in the picture below.

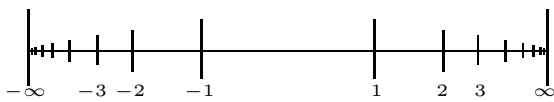


The payoff functions $u_{1}$ and $u_{2}$ are symmetric and $u_{1}$ is given in the table below. Player 1 is the row player, and player 2 is the column player.

\begin{tabular}{c|ccccc|ccccc|}
\hline$u_{1}$ & $-\infty$ & $\ldots$ & -3 & -2 & -1 & 1 & 2 & 3 & $\ldots$ & $\infty$ \\
\hline$\infty$ & 0 & $\ldots$ & 0 & 0 & 0 & 0 & 0 & 0 & $\ldots$ & 0 \\
$\vdots$ & $\vdots$ & $\ddots$ & $\vdots$ & $\vdots$ & $\vdots$ & $\vdots$ & $\vdots$ & $\vdots$ &.$\cdot$ & $\vdots$ \\
-3 & 0 & $\ldots$ & $\frac{1}{3}$ & 0 & 0 & 0 & 0 & $\frac{1}{3}$ & $\ldots$ & 0 \\
-2 & 0 & $\ldots$ & 0 & $\frac{1}{2}$ & 0 & 0 & $\frac{1}{2}$ & 0 & $\ldots$ & 0 \\
-1 & 0 & $\ldots$ & 0 & 0 & 1 & 1 & 0 & 0 & $\ldots$ & 0 \\
1 & 0 & $\ldots$ & 0 & 0 & 0 & 1 & 0 & 0 & $\ldots$ & 0 \\
2 & 0 & $\ldots$ & 0 & 0 & 0 & 0 & $\frac{1}{2}$ & 0 & $\ldots$ & 0 \\
3 & 0 & $\ldots$ & 0 & 0 & 0 & 0 & 0 & $\frac{1}{3}$ & $\ldots$ & 0 \\
$\vdots$ & $\vdots$ &.$\cdot$ & $\vdots$ & $\vdots$ & $\vdots$ & $\vdots$ & $\vdots$ & $\vdots$ & $\ddots$ & $\vdots$ \\
$\infty$ & 0 & $\ldots$ & 0 & 0 & 0 & 0 & 0 & 0 & $\ldots$ & 0 \\
\hline
\end{tabular}

Claim. The action pair $(\infty, \infty)$ is a strongly glof perfect equilibrium. However, $\infty$ is not limit admissible for either player.

Proof. For either player, $\infty$ is not limit admissible because $\infty$ is dominated by every action $k \in \mathbb{Z}$, and every action $k \in \mathbb{N}$ is dominated by action $-k$.

Now, we prove that $\sigma=(\infty, \infty)$ is a strongly glof perfect equilibrium. For every player $i=1,2$ and $k \in \mathbb{N}$, let $B_{i}^{k}=\{-k, \ldots,-1,1, \ldots, k+1\}$ and let $\sigma_{i}^{k}$ be the completely mixed strategy on $B_{i}^{k}$ which assigns probability $\frac{1}{(k+2)^{2}}$ to every action in $B_{i}^{k}$ except action $k+1$ and assigns probability $\left(1-\frac{2 k}{(k+2)^{2}}\right)$ to action $k+1$. It is clear that $\left(B_{i}^{k}\right)_{k=1}^{\infty}$ satisfies the first condition of Definition 4.3. Since $\rho^{w}\left(\sigma_{i}^{k}, \sigma_{i}\right) \rightarrow 0$ for $i=1,2$ as $k \rightarrow \infty$, the second condition also holds. Using the payoff table, one can verify that strategies $(-k-1)$ and $(k+1)$ are the best responses for player 1 against $\sigma_{2}^{k}$ in $A_{1}$. Due to symmetry, the same holds for 
player 2. Thus,

$$
\rho^{s}\left(\sigma_{i}^{k}, B R_{i}\left(\sigma^{k}\right)\right)=1-\left(1-\frac{2 k}{(k+2)^{2}}\right)=\frac{2 k}{(k+2)^{2}},
$$

which tends to zero as $k \rightarrow \infty$. Hence, condition 4 of Definition 4.3 is valid as well, and $\sigma$ is a strongly glof perfect equilibrium.

\section{Relations between definitions of perfect equi- librium}

Simon and Stinchcombe [24] conjectured (on page 1433) that there are strongly perfect equilibria that are not anchored perfect. We provide a concrete example of a completely mixed, and therefore surely strongly perfect, equilibrium that is neither lof perfect nor glof perfect (and therefore also not anchored perfect). Thus, the finitistic approach to perfection does not have a straightforward logical relation to the trembling hand approach.

EXAMPLE 2. The action spaces are $A_{1}=A_{2}=\{1,2,3, \ldots, \infty, \infty+1\}$, where all natural numbers and $\infty+1$ are isolated points, whereas $\infty$ is the limit of the sequence $1,2, \ldots$ The topology is shown in the picture below:

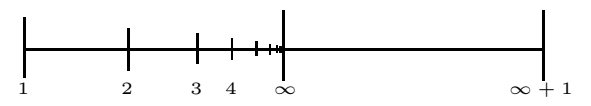

The payoff functions $u_{1}$ and $u_{2}$ are symmetric and $u_{1}$ is given in the table below.

\begin{tabular}{|c|ccccccr|}
\hline$u_{1}$ & 1 & 2 & 3 & 4 & $\ldots$ & $\infty$ & $\infty+1$ \\
\hline 1 & $\frac{4}{8^{2}}$ & $\frac{-1}{8}$ & 0 & 0 & $\ldots$ & 0 & 0 \\
2 & $\frac{4}{16^{2}}$ & 0 & $\frac{-1}{16}$ & 0 & $\ldots$ & 0 & 0 \\
3 & $\frac{4}{32^{2}}$ & 0 & 0 & $\frac{-1}{32}$ & $\ldots$ & 0 & 0 \\
4 & $\frac{4}{64^{2}}$ & 0 & 0 & 0 & $\ldots$ & 0 & 0 \\
$\vdots$ & $\vdots$ & $\vdots$ & $\vdots$ & $\vdots$ & $\ddots$ & $\vdots$ & $\vdots$ \\
$\infty$ & 0 & 0 & 0 & 0 & $\ldots$ & 0 & 0 \\
$\infty+1$ & 0 & 0 & 0 & 0 & $\ldots$ & 0 & 0 \\
\hline
\end{tabular}


Define the strategies $\sigma_{1}$ and $\sigma_{2}$ by

$$
\sigma_{1}=\sigma_{2}=\frac{1}{4} \cdot \delta(1)+\frac{1}{8} \cdot \delta(2)+\frac{1}{16} \cdot \delta(3)+\ldots+\frac{1}{4} \cdot \delta(\infty)+\frac{1}{4} \cdot \delta(\infty+1) .
$$

Claim. The strategy profile $\left(\sigma_{1}, \sigma_{2}\right)$ is a completely mixed, and hence strongly perfect equilibrium. However, it is neither lof perfect nor weakly glof perfect. This verifies the conjecture of S\&S [24] ${ }^{6}$.

Proof. It is clear that $\left(\sigma_{1}, \sigma_{2}\right)$ is a completely mixed Nash equilibrium. Hence, it is a strongly perfect equilibrium.

We show that the completely mixed equilibrium is not lof perfect. Suppose by way of contradiction that $\left(\sigma_{1}, \sigma_{2}\right)$ is lof perfect. For every player $i$, take a sequence $\left(B_{i}^{k}\right)_{k=1}^{\infty}$ of finite subsets of $A_{i}$ and a sequence of strategies $\left(\sigma_{i}^{k}\right)_{k=1}^{\infty}$ that satisfy the conditions in Definition 4.2. Let $m_{k}$ and $n_{k}$ be the largest positive integers in the sets $B_{1}^{k}$ and $B_{2}^{k}$, respectively. Since $\infty+1$ is an isolated point of the action sets, for large $k$ each $B_{i}^{k}$ includes $\infty+1$. Moreover, for large $k, \infty+1$ must be a best response for player 1 against $\sigma_{2}^{k}$ among $B_{1}^{k}$, because $\sigma_{1}$ places positive probability on $\infty+1$. As strategy $\infty+1$ has payoff zero for player 1 , for large $k$ player 1 cannot have an action in $B_{1}^{k}$ which gives him a positive payoff against $\sigma_{2}^{k}$. Since player 1 would get a positive payoff for every action $m \geq n_{k}$, we have $m_{k}<n_{k}$. Then also $n_{k}<m_{k}$ by symmetry, which is a contradiction. By similar arguments the completely mixed equilibrium is not weakly glof either.

We continue with a discussion of the relations between the different approaches to finitistic perfect equilibrium.

Proposition 6.1 Every strongly glof perfect equilibrium is lof perfect.

Proof. Suppose that $\sigma=\left(\sigma_{i}\right)_{i \in N}$ is a strongly glof perfect equilibrium. Then, for every $i \in N$, there is a sequence $\left(B_{i}^{k}\right)_{k=1}^{\infty}$ of finite subsets of $A_{i}$ and a sequence of strategies $\left(\sigma_{i}^{k}\right)_{k=1}^{\infty}$ that satisfy the conditions in Definition 4.3. We show that they also satisfy condition 3 of Definition 4.2 . Since for every $i$, we have $\rho^{s}\left(\sigma_{i}^{k}, B R_{i}\left(\sigma^{k}\right)\right) \rightarrow 0$ as $k \rightarrow \infty$, there exists a sequence $\left(\mu_{i}^{k}\right)_{k=1}^{\infty}$ such that $\mu_{i}^{k} \in B R_{i}\left(\sigma^{k}\right)$ for every $k \in \mathbb{N}$ and $\rho^{s}\left(\sigma_{i}^{k}, \mu_{i}^{k}\right) \rightarrow 0$ as $k \rightarrow \infty$. As $u_{i}$ is

\footnotetext{
${ }^{6}$ Their conjecture only concerns anchored perfect equilibrium. We show the stronger claim that the completely mixed equilibrium is even not a lof perfect equilibrium.
} 
a continuous function, $P B R_{i}\left(\sigma^{k}\right)$ is a closed, hence Borel, subset of the action space $A_{i}$. Moreover, since best responses put weight 1 on pure best responses,

$$
\rho^{s}\left(\sigma_{i}^{k}, \mu_{i}^{k}\right) \geq\left|\sigma_{i}^{k}\left(P B R_{i}\left(\sigma^{k}\right)\right)-\mu_{i}^{k}\left(P B R_{i}\left(\sigma^{k}\right)\right)\right|=\left|\sigma_{i}^{k}\left(P B R_{i}\left(\sigma^{k}\right)\right)-1\right|
$$

for every $i$ and $k$. So, $\sigma_{i}^{k}\left(P B R_{i}\left(\sigma^{k}\right)\right) \rightarrow 1$ as $k \rightarrow \infty$. This implies that for large $k$, player $i$ has a pure best response to $\sigma^{k}$ which lies in $B_{i}^{k}$. Consequently, $\sigma_{i}^{k}\left(P B R_{i}\left(\sigma^{k} \mid B_{i}^{k}\right)\right) \rightarrow 1$ as $k \rightarrow \infty$, and $\sigma$ is a lof perfect equilibrium.

Theorem 5.3 shows that the lof perfect equilibrium in Example 2.4 in S\&S is not weakly glof perfect (and hence not strongly glof perfect either). Next we present an example of a weakly glof perfect equilibrium that is not lof perfect. Consequently, lof perfection and weak glof perfection are not comparable.

EXAMPLE 3. In this game the action spaces are

$$
A_{1}=A_{2}=\{-1,-2,-3, \ldots, 0, \ldots, 3,2,1\},
$$

where all integers except 0 are isolated points, whereas 0 is the limit point of the sequences $-1,-2, \ldots$ and $1,2, \ldots$ The topological structure is shown in the picture below.

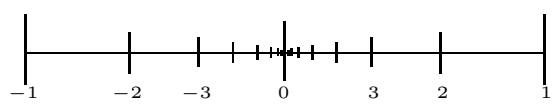

The topology can be metrized by the following metric. For every $k, l \in A_{i}$,

$$
d(k, l)= \begin{cases}0 & \text { if } k=l=0 \\ \left|\frac{1}{l}\right| & \text { if } k=0 \text { and } l \neq 0 \\ \left|\frac{1}{k}\right| & \text { if } k \neq 0 \text { and } l=0 \\ \left|\frac{1}{k}-\frac{1}{l}\right| & \text { if } k \neq 0 \text { and } l \neq 0 .\end{cases}
$$

The payoff functions $u_{1}$ and $u_{2}$ are symmetric and $u_{1}$ is given in the table below. 


\begin{tabular}{|c|cccc|c|cccc|}
\hline$u_{1}$ & -1 & -2 & -3 & $\cdots$ & 0 & $\cdots$ & +3 & +2 & +1 \\
\hline-1 & 1 & $\frac{1}{2}$ & $\frac{1}{3}$ & $\cdots$ & 0 & $\cdots$ & 0 & 0 & 1 \\
-2 & 0 & 0 & 0 & $\cdots$ & 0 & $\cdots$ & 0 & $\frac{1}{2}$ & 0 \\
-3 & 0 & 0 & 0 & $\cdots$ & 0 & $\cdots$ & $\frac{1}{3}$ & 0 & 0 \\
$\vdots$ & $\vdots$ & $\vdots$ & $\vdots$ & $\ddots$ & $\vdots$ & $\cdot$ & $\vdots$ & $\vdots$ & $\vdots$ \\
0 & 0 & 0 & 0 & $\cdots$ & 0 & $\cdots$ & 0 & 0 & 0 \\
\hline$\vdots$ & $\vdots$ & $\vdots$ & $\vdots$ & $\cdot$ & $\vdots$ & $\ddots$ & $\vdots$ & $\vdots$ & $\vdots$ \\
+3 & 0 & 0 & 0 & $\cdots$ & 0 & $\cdots$ & 0 & 0 & 0 \\
+2 & 0 & 0 & 0 & $\cdots$ & 0 & $\cdots$ & 0 & 0 & 0 \\
+1 & 0 & 0 & 0 & $\cdots$ & 0 & $\cdots$ & 0 & 0 & 0 \\
\hline
\end{tabular}

Claim. The Nash equilibrium $\left(\sigma_{1}, \sigma_{2}\right)=(0,0)$ is weakly glof perfect. However, $\left(\sigma_{1}, \sigma_{2}\right)$ is not lof perfect, and therefore by Proposition 6.1 not strongly glof perfect either.

Proof. We show that $\left(\sigma_{1}, \sigma_{2}\right)$ is a weakly glof perfect. For every $k \in \mathbb{N}$, define $\varepsilon_{k}=\frac{1}{k(k+3)}$. Moreover, for $k \in \mathbb{N}$ and $i=1,2$, write

$$
B_{i}^{k}=\{-1,-2, \ldots,-k\} \bigcup\{k, \ldots, 2,1\} .
$$

Let $\sigma_{i}^{k}$ be the completely mixed strategy on $B_{i}^{k}$ that assigns probability $\varepsilon_{k}$ to every action in $B_{i}^{k}$ except action $k$, to which it assigns probability $1-(2 k-1) \varepsilon_{k}$. Note that due to the choice of $\varepsilon_{k}$, we have $1-(2 k-1) \varepsilon_{k}>\varepsilon_{k}>0$. It is clear that conditions 1 and 2 of Definition 4.3 are satisfied. It remains to verify that condition 5 holds as well. According to the payoff table, player 1's pure best response to $\sigma_{2}^{k}$ is either -1 or $-k$. Since for every $k \geq 2$ we have

$$
\begin{aligned}
u_{1}\left(\delta(-1), \sigma_{2}^{k}\right) & =\varepsilon_{k}\left(1+\frac{1}{2}+\frac{1}{3}+\ldots+\frac{1}{k}+1\right) \\
& \leq \varepsilon_{k}(k+1) \\
& <\left(1-(2 k-1) \varepsilon_{k}\right) \frac{1}{k} \\
& =u_{1}\left(\delta(-k), \sigma_{2}^{k}\right),
\end{aligned}
$$


player 1's unique best response against $\sigma_{2}^{k}$ is $-k$. We prove that

$$
\rho^{w}\left(\sigma_{1}^{k}, B R_{1}\left(\sigma^{k}\right)\right)=\rho^{w}\left(\sigma_{1}^{k}, \delta(-k)\right) \leq \frac{2}{k} .
$$

Let $\varepsilon>\frac{2}{k}=d(-k, k)$. It is easy to see that $(2 k-1) \varepsilon_{k} \leq \varepsilon$. Take an arbitrary set $B \subseteq A_{1}$.

A. We show that $\delta(-k)(B) \leq \sigma_{1}^{k}\left(B^{\varepsilon}\right)+\varepsilon$. If $-k \notin B$. Then

$$
\delta(-k)(B)=0 \leq \sigma_{1}^{k}\left(B^{\varepsilon}\right)+\varepsilon
$$

If $-k \in B$. Then $k \in B^{\varepsilon}$. So

$$
\delta(-k)(B)=1 \leq\left(1-(2 k-1) \varepsilon_{k}\right)+\varepsilon=\sigma_{1}^{k}(k)+\varepsilon \leq \sigma_{1}^{k}\left(B^{\varepsilon}\right)+\varepsilon .
$$

B. We show that $\sigma_{1}^{k}(B) \leq \delta(-k)\left(B^{\varepsilon}\right)+\varepsilon$. If $-k \in B$. Then

$$
\sigma_{1}^{k}(B) \leq 1<1+\varepsilon=\delta(-k)\left(B^{\varepsilon}\right)+\varepsilon
$$

If $-k \notin B$ and $k \notin B$. Then

$$
\sigma_{1}^{k}(B) \leq(2 k-1) \varepsilon_{k} \leq \varepsilon \leq \delta(-k)\left(B^{\varepsilon}\right)+\varepsilon .
$$

If $-k \notin B$ and $k \in B$. Then $-k \in B^{\varepsilon}$. So

$$
\sigma_{1}^{k}(B) \leq 1+\varepsilon=\delta(-k)\left(B^{\varepsilon}\right)+\varepsilon
$$

So, by $\mathbf{A}, \mathbf{B}$, and the choice of $\varepsilon, \rho^{w}\left(\sigma_{1}^{k}, \delta(-k)\right) \leq \frac{2}{k}$. Since the payoff functions are symmetric this argument is also valid for the second player. Therefore, condition 5 of Definition 4.3 holds and $\sigma$ is a weakly glof perfect equilibrium as claimed.

We show that $(0,0)$ is not lof perfect. Suppose the opposite. Then by Definition 4.2 for each $i=1,2$ there is non-empty finite subset $B_{i}$ of $A_{i}$ and a completely mixed strategy $\sigma_{i}$ in $\Delta\left(B_{i}\right)$ such that

$$
\sigma_{i}\left(\operatorname{PBR}_{i}\left(\sigma \mid B_{i}\right)\right) \geq \frac{2}{3}
$$

where $\sigma=\left(\sigma_{1}, \sigma_{2}\right)$. Since -1 is an isolated point, w.l.o.g. $-1 \in B_{i}$ for each $i=1,2$. Furthermore, $\operatorname{PBR}_{i}\left(\sigma \mid B_{i}\right)$ is a subset of $\{-1,-2, \ldots\}$. Define

$$
M_{i}=-\min \left\{x \mid x \in \operatorname{PBR}_{i}\left(\sigma \mid B_{i}\right)\right\} \quad \text { for } i=1,2
$$


Assume w.l.o.g. that $M_{1} \geq M_{2}$. Because $-M_{1} \in P B R_{1}\left(\sigma \mid B_{1}\right)$ and $-1 \in B_{1}$ we have

$$
u_{1}\left(\delta(-1), \sigma_{2}\right) \leq u_{1}\left(\delta\left(-M_{1}\right), \sigma_{2}\right)
$$

Thus, according to the payoff table,

$$
\frac{2}{3} \cdot \frac{1}{M_{2}} \leq u_{1}\left(\delta(-1), \sigma_{2}\right) \leq u_{1}\left(\delta\left(-M_{1}\right), \sigma_{2}\right) \leq \frac{1}{3} \cdot \frac{1}{M_{1}}
$$

Rewriting yields $\frac{M_{2}}{2} \geq M_{1}$. Consequently $\frac{M_{2}}{2} \geq M_{2}$. Contradiction.

Finally, in this section we present a modification of the example in S\&S of a pure equilibrium that is limit of finite, while one of the players plays an action that is not limit admissible. Theorem 5.3 shows that the equilibrium is not weakly glof.

EXAMPLE 4 The game has two players with action spaces $A_{1}=A_{2}=[0,1]$ and utility functions $u_{1}=0$ and

$$
u_{2}\left(a_{1}, a_{2}\right)= \begin{cases}\left(a_{2}-1\right) \cdot a_{1} & \text { if } a_{1} \leq a_{2} \\ \left(a_{2}+1\right) \cdot a_{1}-2 a_{2} & \text { if } a_{1} \geq a_{2}\end{cases}
$$

In the following picture the function $a_{1} \mapsto u_{2}\left(a_{1}, a_{2}\right)$ is depicted for a fixed action $a_{2}$ of player 2 .

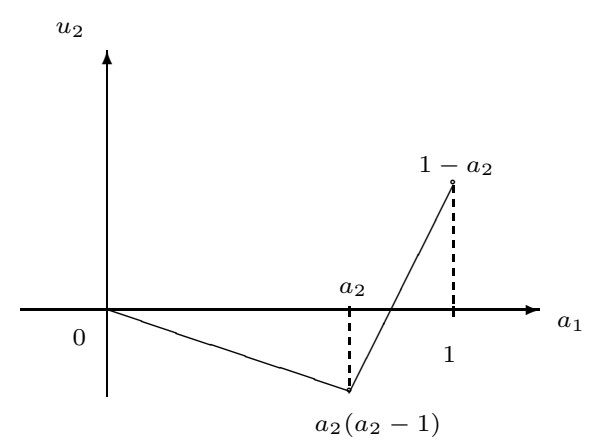

Claim. The action pair $(0,1)$ is a lof perfect equilibrium. However, $a_{2}=0$ is the unique dominant strategy, so that $a_{2}=1$ is not limit admissible. Consequently, the equilibrium is not weakly glof perfect.

Proof. The strategy $a_{2}=0$ is the unique dominant strategy for player 2, so that $a_{2}=1$ is not limit admissible. Hence, by Theorem 5.3 , the pair $(0,1)$ is not weakly glof perfect. 
In order to show that $(0,1)$ is lof perfect, take $\varepsilon \in(0,1)$. For $n \in \mathbb{N}$, consider the finite subsets

$$
B_{1}^{n}=\left\{\frac{i}{n} \mid i=0,1, \ldots, n\right\} \quad \text { and } \quad B_{2}^{n}=\left\{\frac{i}{n} \mid i=1, \ldots, n\right\}
$$

of $A_{1}$ and $A_{2}$, respectively. For $0<s<\frac{\varepsilon}{1+n^{2}}$, define the strategy

$$
\tau_{1}(\varepsilon, s)=(1-\varepsilon) \delta(0)+(\varepsilon-s) \delta\left(\frac{1}{n}\right)+\frac{s}{n-1} \sum_{i=2}^{n} \delta\left(\frac{i}{n}\right) .
$$

Then for every $a_{2}^{j}=\frac{j}{n}, j=1, \ldots, n-1$ we have

$$
\begin{aligned}
u_{2}\left(\tau_{1}(\varepsilon, s), \delta\left(a_{2}^{j}\right)\right) & =(\varepsilon-s) \cdot u_{2}\left(\frac{1}{n}, \frac{j}{n}\right)+\frac{s}{n-1} \cdot\left[\sum_{i=2}^{n} u_{2}\left(\frac{i}{n}, \frac{j}{n}\right)\right] \\
& \leq(\varepsilon-s) \cdot u_{2}\left(\frac{1}{n}, \frac{j}{n}\right)+\frac{s}{n-1} \cdot\left[\sum_{i=2}^{n} 1\right] \\
& \leq(\varepsilon-s) \cdot\left(\frac{1}{n}\right) \cdot\left(\frac{j}{n}-1\right)+s \\
& \leq(\varepsilon-s)\left(\frac{1}{n}\right)\left(-\frac{1}{n}\right)+s \\
& =s \cdot \frac{1+n^{2}}{n^{2}}-\frac{\varepsilon}{n^{2}} \\
& <0 .
\end{aligned}
$$

This outcome is less than zero, but player 2 can get zero by choosing $a_{2}^{n}=1$ in the set $B_{2}^{n}$. Hence, action pair $(0,1)$ is a lof perfect equilibrium.

\section{Two players}

For finite strategic form games it is well-known that perfection implies undominatedness. A similarly well-known theorem of van Damme (see for example van Damme [8], Theorem 3.2.2) states that for bimatrix games the converse implication also holds.

Theorem 7.1 Let $(p, q)$ be a strategy pair of the bimatrix game $(A, B)$. Equivalent are

[1] The strategy pair $(p, q)$ is a perfect equilibrium.

[2] The strategy pair $(p, q)$ is a Nash equilibrium and both $p$ and $q$ are undominated strategies.

Theorem 5.1 and Proposition 5.2 show that, in the appropriately adjusted sense, the implication from (1) to (2) is also valid in the setting with infinite compact 
action spaces. A natural guess would be that the appropriately adjusted converse would also be true for two-player infinite games. However, the following example shows that the converse implication from (2) to (1) no longer holds for games with infinite action spaces. (In their Example 2 Méndez et al. [15] address the same issue in a game with interval action spaces.)

EXAMPLE 5. The action spaces are $A_{1}=\{T, B\}$ and $A_{2}=\{1,2,3,4, \ldots, \infty\}$, where all natural numbers are isolated points, whereas $\infty$ is the limit point of the sequence $1,2,3, \ldots$ The payoffs for the players are given by

$$
u_{1}=\left[\begin{array}{lllll}
0 & 0 & 0 & \ldots & 0 \\
1 & 1 & 1 & \ldots & 1
\end{array}\right] \text { and } u_{2}=\left[\begin{array}{rrrrrr}
0 & \frac{1}{2} & \frac{1}{3} & \frac{1}{4} & \ldots & 0 \\
0 & -\frac{1}{4} & -\frac{1}{9} & -\frac{1}{16} & \ldots \ldots & 0
\end{array}\right]
$$

Claim. The action pair $(B, 1)$ is a Nash equilibrium in undominated strategies, yet $(B, 1)$ is not a weakly perfect equilibrium.

Proof. Clearly $(B, 1)$ is a Nash equilibrium and actions $B$ and 1 are undominated in $A_{1}$ and $A_{2}$ respectively.

We prove that the action pair $\sigma=(B, 1)$ is not a weakly perfect equilibrium. Take any completely mixed strategy $\tau_{1}=(p, 1-p)$ of player 1 . Take a natural number $k>\frac{1-p}{p}$. Then $\frac{1-p}{k}<p$, so that

$$
\begin{aligned}
u_{2}\left(\tau_{1}, \delta(k)\right) & =p \cdot \frac{1}{k}-(1-p) \cdot \frac{1}{k^{2}} \\
& =\frac{1}{k} \cdot\left[p-\frac{1-p}{k}\right] \\
& >0 .
\end{aligned}
$$

Since $u_{2}\left(\tau_{1}, \delta(1)\right)=0$, this implies that $\delta(1)$ is not a best response for player 2 against any completely mixed strategy of player 1 . Hence, $\sigma=(B, 1)$ is not a weakly perfect equilibrium.

Note that the above example is in a very strong sense minimal. The action space $A_{2}$ is in a very natural way the "smallest" example of a compact set that is not finite. And also $A_{1}$ is minimal in the sense that it is the smallest example of an action space in which player 1 actually has a genuine choice to make. 


\section{Invariance}

Another remarkable feature of the game in Example 5 is that $(B, \infty)$ is a strongly (and hence also weakly) perfect equilibrium. This is surprising in the sense that $\infty$ is payoff equivalent to 1 for player 2 . Thus, both strong and weak perfection violate invariance in this setting, while perfect equilibrium satisfies invariance in the setting of finite strategic form games (see for example Mertens [18] and Vermeulen and Jansen [25]).

The underlying cause of this seeming breakdown of invariance is that in the finite case the space of actions is, from a topological perspective, a very simple object. All actions are isolated points, and hence there is a homeomorphism that transforms one into the other while the induced transformation on payoffs equals the identity in the case of payoff equivalence.

However, in the infinite case two actions giving the same payoff might be totally different from a topological perspective. Therefore, only conditional on having a homeomorphism that preserves payoffs, we can conclude that strong and weak perfection are invariant too. This result is stated in the following theorem.

Theorem 8.1 Both strongly and weakly perfect equilibrium are invariant under payoff preserving homeomorphic transformations of the action spaces.

Note that, for finite games, invariance with respect to payoff preserving homeomorphic transformations is in fact equivalent to invariance. Hence, the above Theorem implies that for finite strategic form games perfect equilibrium is invariant.

\section{Discussion}

We studied the relations between various generalizations of the basic notion of perfect equilibrium to the context of strategic form games with compact action spaces and continuous payoff functions. We showed the existence of weakly glof perfect equilibrium, and presented several examples of games with compact action spaces that clarify the relationships between admissibility, trembling hand perfect equilibrium, and finitistic perfect equilibrium. 
Specifically, we showed in Example 5 that the equivalence between perfection and undominatedness for bimatrix games no longer holds for general two-person strategic form games with compact action spaces and continuous payoffs.

We also verified a conjecture of Simon and Stichcombe [24] with an example of a completely mixed Nash equilibrium that is not perfect in any of the finitistic definitions. Despite its appeal to basic intuition of the finitistic approach, our results (especially Examples 1 and 2) seem to imply a severe critique on the finitistic approach. Example 1 shows that finitistic perfection may easily fail to be limit admissible, a fact that was already observed by Simon and Stinchcombe for lof perfection. Example 2 shows that a completely mixed equilibrium need not be perfect in any of the finitistic approaches.

In Example 2 the completely mixed equilibrium is part of a component of Nash equilibria, one of which is still finitistically perfect. It remains an open question whether an isolated completely mixed equilibrium is automatically perfect in the finitistic approach. Another possible question for future research is the existence of strongly glof perfect equilibrium.

\section{A Proof of Theorem 4.4}

The proof of Theorem 4.4 is based on the fixed point Theorem of Kakutani. We state the Theorem of Kakutani first.

Let $X$ and $Y$ be two metric spaces. A correspondence between $X$ and $Y$ is a map from the elements of $X$ to the collection of all subsets of $Y$. So, for every $x \in X$, we have $F(x) \subseteq Y$. A correspondence $F$ between $X$ and $Y$ is upperhemicontinuous if for every $x \in X$ and every sequence $\left(x_{n}\right)_{n=1}^{\infty}$ in $X$ converging to $x$ and every sequence $\left(y_{n}\right)_{n=1}^{\infty}$ in $Y$ converging to $y$ with $y_{n} \in F\left(x_{n}\right)$ it holds that $y \in F(x)$. A point $x \in X$ is called a fixed point for a correspondence $F: X \rightarrow X$ when $x \in F(x)$.

Theorem A.1 (Kakutani's fixed point theorem) Let $X$ be a non-empty, compact and convex subset of an Euclidean space. Let $F: X \rightarrow X$ be an upperhemicontinuous correspondence such that $F(x)$ is non-empty, closed and convex for all $x \in X$. Then $F$ has a fixed point. 
We show Theorem 4.4. W.l.o.g. suppose that $A_{i} \subseteq[0,1]$ for every $i \in N$. Fix $k \in \mathbb{N}$. For every $i$, construct a finite subset $B_{i}^{k}$ of $A_{i}$ with $\left|B_{i}^{k}\right| \leq 3 k$ and for every $a \in A_{i}$ there is a $b \in B_{i}^{k}$ such that $|a-b| \leq \frac{1}{k}$. Write $n_{i k}=\left|B_{i}^{k}\right|$ and $\varepsilon=\frac{1}{3 k^{2}}$. For every $i$, define

$$
\Delta_{i}^{k}(\varepsilon)=\left\{\nu_{i} \in \Delta\left(B_{i}^{k}\right) \mid \nu_{i}(b) \geq \varepsilon \text { for every } b \in B_{i}^{k}\right\}
$$

and $\Delta^{k}(\varepsilon)=\prod_{i \in N} \Delta_{i}^{k}(\varepsilon)$. Define the correspondence $A B R_{i}^{k}: \Delta^{k}(\varepsilon) \rightarrow \Delta_{i}^{k}(\varepsilon)$ by

$$
A B R_{i}^{k}(\sigma)=\left\{\mu_{i} \in \Delta_{i}^{k}(\epsilon) \mid \rho^{w}\left(\mu_{i}, B R_{i}(\sigma)\right) \leq \frac{2}{k}\right\}
$$

for every $\sigma=\left(\sigma_{i}\right)_{i \in N} \in \Delta^{k}(\varepsilon)^{7}$. Furthermore, define the correspondence $A B R^{k}: \Delta^{k}(\varepsilon) \rightarrow \Delta^{k}(\varepsilon)$ by, for every $\sigma \in \Delta(A)$,

$$
A B R^{k}(\sigma)=\prod_{i \in N} A B R_{i}^{k}(\sigma) .
$$

We verify the conditions of Theorem A.1.

A. Clearly $\Delta^{k}(\varepsilon)$ is compact and convex. Further, since $\varepsilon=\frac{1}{3 k^{2}}$ and $\left|B_{i}^{k}\right| \leq$ $3 k$, the set $\Delta^{k}(\varepsilon)$ is not empty.

B. We verify that $A B R^{k}(\sigma)$ satisfies the conditions of the Theorem of Kakutani.

B1. We show for every $i \in N$ that $A B R_{i}^{k}(\sigma)$ is non-empty. Take $a \in P B R_{i}(\sigma)$. Then, there is a $b \in B_{i}^{k}$ such that $|a-b| \leq \frac{1}{k}$. Hence, $\rho^{w}(\delta(a), \delta(b)) \leq \frac{1}{k}$. Now, for $x \in B_{i}^{k}$ define

$$
\mu_{i}(x)= \begin{cases}\varepsilon & \text { if } x \neq b \\ 1-\left(n_{i k}-1\right) \epsilon & \text { if } x=b .\end{cases}
$$

Then, $\mu_{i} \in \Delta_{i}^{k}(\varepsilon)$. Moreover,

$$
\rho^{w}\left(\mu_{i}, \delta(b)\right) \leq\left\|\mu_{i}-\delta(b)\right\|_{\infty}=\left(n_{i k}-1\right) \varepsilon \leq 3 k \cdot \frac{1}{3 k^{2}}=\frac{1}{k} .
$$

Then, by the triangle inequality we have

$$
\rho^{w}\left(\mu_{i}, \delta(a)\right) \leq \frac{1}{k}+\frac{1}{k}=\frac{2}{k} .
$$

Thus, $\rho^{w}\left(\mu_{i}, B R_{i}(\sigma)\right) \leq \frac{2}{k}$, which implies $\mu_{i} \in A B R_{i}^{k}(\sigma)$. Hence, $A B R^{k}(\sigma)$ is non-empty.

\footnotetext{
${ }^{7} A B R$ stands for approximate best response.
} 
B2. Clearly, for every $i, A B R_{i}^{k}(\sigma)$ is closed and therefore $A B R^{k}(\sigma)$ is closed as well.

B3. We prove that $A B R_{i}^{k}(\sigma)$ is convex for every $i$. Take strategies $\mu, \nu \in$ $A B R_{i}^{k}(\sigma)$. We prove that $c \mu+(1-c) \nu \in A B R_{i}^{k}(\sigma)$ for every $c \in[0,1]$. Since $\mu, \nu \in A B R_{i}^{k}(\sigma)$, we have $\rho^{w}\left(\mu, B R_{i}(\sigma)\right) \leq \frac{2}{k}$ and $\rho^{w}\left(\nu, B R_{i}(\sigma)\right) \leq \frac{2}{k}$. So there are strategies $\mu^{\prime}, \nu^{\prime} \in B R_{i}(\sigma)$ such that

$$
\rho^{w}\left(\mu, \mu^{\prime}\right) \leq \frac{2}{k} \quad \text { and } \quad \rho^{w}\left(\nu, \nu^{\prime}\right) \leq \frac{2}{k} .
$$

Take an arbitrary $\delta>\frac{2}{k}$. Then, for every non-empty measurable set $A \subseteq A_{i}$, we have

$$
\begin{aligned}
& \mu(A) \leq \mu^{\prime}\left(A^{\delta}\right)+\delta \\
& \mu^{\prime}(A) \leq \mu\left(A^{\delta}\right)+\delta
\end{aligned}
$$

and

$$
\begin{gathered}
\nu(A) \leq \nu^{\prime}\left(A^{\delta}\right)+\delta \\
\nu^{\prime}(A) \leq \nu\left(A^{\delta}\right)+\delta .
\end{gathered}
$$

Then,

$$
\begin{gathered}
c \mu(A)+(1-c) \nu(A) \leq c \mu^{\prime}\left(A^{\delta}\right)+(1-c) \nu^{\prime}\left(A^{\delta}\right)+\delta \\
c \mu^{\prime}(A)+(1-c) \nu^{\prime}(A) \leq c \mu\left(A^{\delta}\right)+(1-c) \nu\left(A^{\delta}\right)+\delta .
\end{gathered}
$$

Hence,

$$
\rho^{w}\left(c \mu+(1-c) \nu, c \mu^{\prime}+(1-c) \nu^{\prime}\right) \leq \delta,
$$

which by the choice of $\delta$ implies

$$
\rho^{w}\left(c \mu+(1-c) \nu, c \mu^{\prime}+(1-c) \nu^{\prime}\right) \leq \frac{2}{k} .
$$

Since $B R_{i}(\sigma)$ is a convex set, $c \mu^{\prime}+(1-c) \nu^{\prime} \in B R_{i}(\sigma)$. Thus,

$$
\rho^{w}\left(c \mu+(1-c) \nu, B R_{i}(\sigma)\right) \leq \frac{2}{k}
$$

which implies $c \mu+(1-c) \nu \in A B R_{i}^{k}(\sigma)$.

C. We show that $A B R^{k}$ is upper-hemicontinuous. Take sequences $\left(\sigma^{m}\right)_{m=1}^{\infty}$ and $\left(\tau^{m}\right)_{m=1}^{\infty}$ in $\Delta^{k}(\epsilon)$ such that $\lim _{m \rightarrow \infty} \sigma^{m}=\sigma$ and $\lim _{m \rightarrow \infty} \tau^{m}=\tau$ in Euclidean distance, and such that $\tau^{m} \in A B R^{k}\left(\sigma^{m}\right)$ for every $m \in \mathbb{N}$. We show $\tau_{i} \in$ $A B R_{i}^{k}(\sigma)$ for every $i \in N$, so that $\tau \in A B R^{k}(\sigma)$. 
Take $m \in \mathbb{N}$. Since $\tau_{i}^{m} \in A B R_{i}^{k}\left(\sigma^{m}\right)$, we have $\rho^{w}\left(\tau_{i}^{m}, B R_{i}\left(\sigma^{m}\right)\right) \leq \frac{2}{k}$. As $B R_{i}\left(\sigma^{m}\right)$ is a closed set with respect to the weak metric, there is a strategy $\mu_{i}^{m} \in B R_{i}\left(\sigma^{m}\right)$ such that $\rho^{w}\left(\tau_{i}^{m}, \mu_{i}^{m}\right) \leq \frac{2}{k}$. Because $\Delta\left(A_{i}\right)$ is sequentially compact with respect to the weak metric, the sequence $\left(\mu_{i}^{m}\right)_{m=1}^{\infty}$ has a subsequence $\left(\mu_{i}^{m_{\ell}}\right)_{\ell=1}^{\infty}$ with $\rho^{w}\left(\mu_{i}^{m_{\ell}}, \mu_{i}\right) \rightarrow 0$ as $l \rightarrow \infty$ for some $\mu_{i} \in \Delta\left(A_{i}\right)$. Then, by continuity of $u_{i}$ with respect to $\rho^{w}$, we have $\mu_{i} \in B R_{i}(\sigma)$. Moreover, $\rho^{w}\left(\tau_{i}, \mu_{i}\right) \leq \frac{2}{k}$. Hence, $\rho^{w}\left(\tau_{i}, B R_{i}(\sigma)\right) \leq \frac{2}{k}$. This shows that the correspondence $A B R^{k}$ is upperhemicontinuous. This concludes $\mathbf{C}$.

By $\mathbf{A}, \mathbf{B}$, and $\mathbf{C}$ we know that each $A B R^{k}$ satisfies the conditions of Theorem A.1. Hence, for every $k$, there is a $\sigma^{k} \in \Delta^{k}(\varepsilon)$ such that $\sigma^{k} \in A B R^{k}\left(\sigma^{k}\right)$, which means that $\sigma_{i}^{k} \in A B R_{i}^{k}\left(\sigma^{k}\right)$ for every $i$. Hence, $\rho^{w}\left(\sigma_{i}^{k}, B R_{i}\left(\sigma^{k}\right)\right) \leq \frac{2}{k}$ for every $i$. Since $\Delta(A)$ is sequentially compact with respect to the weak metric, we can assume w.l.o.g. that the sequence $\sigma^{k}=\left(\sigma_{i}^{k}\right)_{i \in N}$ converges to a strategy profile $\sigma=\left(\sigma_{i}\right)_{i \in N}$ when $k \rightarrow \infty$. Note that $\rho^{w}\left(\sigma_{i}^{k}, B R_{i}\left(\sigma^{k}\right)\right) \rightarrow 0$ as $k \rightarrow \infty$. Hence, $\sigma=\left(\sigma_{i}\right)_{i \in N}$ is a weakly glof perfect equilibrium.

It remains to show that every weakly glof perfect equilibrium is a Nash equilibrium. Suppose that $\sigma=\left(\sigma_{i}\right)_{i \in N}$ is a weakly glof perfect equilibrium. Then, by definition, for every $i$ there is a sequence $\left(\sigma_{i}^{k}\right)_{k=1}^{\infty}$ such that $\rho^{w}\left(\sigma_{i}^{k}, \sigma_{i}\right) \rightarrow 0$ and $\rho^{w}\left(\sigma_{i}^{k}, B R_{i}\left(\sigma^{k}\right)\right) \rightarrow 0$ as $k \rightarrow \infty$. Therefore, for every $i$ there is also a sequence $\left(\mu_{i}^{k}\right)_{k=1}^{\infty}$ such that $\mu_{i}^{k} \in B R_{i}\left(\sigma^{k}\right)$ for every $k \in \mathbb{N}$ and $\rho^{w}\left(\sigma_{i}^{k}, \mu_{i}^{k}\right) \rightarrow 0$ as $k \rightarrow \infty$. By the triangle inequality for $\rho^{w}$, this implies that for every $i, \rho^{w}\left(\mu_{i}^{k}, \sigma_{i}\right) \rightarrow 0$ as $k \rightarrow \infty$. Hence, again by continuity of $u_{i}$, we obtain $\sigma_{i} \in B R_{i}(\sigma)$ for every $i \in N$, as desired.

\section{B Proofs for Section 5}

Lemma B.1 Suppose that $\tau_{i}$ dominates $\sigma_{i}$. Then there is an $a_{-i} \in A_{-i}$ such that $u_{i}\left(a_{-i}, \tau_{i}\right)>u_{i}\left(a_{-i}, \sigma_{i}\right)$.

Proof. Suppose that $u_{i}\left(a_{-i}, \tau_{i}\right)=u_{i}\left(a_{-i}, \sigma_{i}\right)$ for every $a_{-i} \in A_{-i}$. Then, for every $\sigma_{-i} \in \Delta\left(A_{-i}\right)$ we have $u_{i}\left(\sigma_{-i}, \tau_{i}\right)=u_{i}\left(\sigma_{-i}, \sigma_{i}\right)$. Contradiction.

Lemma B.2 Suppose that strategy $\tau_{i} \in \Delta\left(A_{i}\right)$ dominates $\sigma_{i} \in \Delta\left(A_{i}\right)$. Then

$$
u_{i}\left(\sigma \mid \tau_{i}\right)>u_{i}\left(\sigma \mid \sigma_{i}\right)
$$


for any completely mixed strategy profile $\sigma \in \Delta(A)$. Consequently, any best response to a completely mixed strategy profile is undominated.

Proof. This is a direct consequence of Lemma B.1 and the continuity of the payoff function $u_{i}$ on $\Delta(A)$.

The next Theorem is a direct consequence of the monotonicity of measure.

Lemma B.3 Suppose that $f$ is a real function on $X$ which is strictly positive and $\mu$ is a measure on $X$ such that $\mu(X)>0$. Then, $\int_{X} f d \mu>0$.

Proof. Let $F_{n}=\left\{x \in X \mid f(x) \geq \frac{1}{n}\right\}$. It is clear that $F_{1} \subseteq F_{2} \subseteq F_{3} \subseteq \cdots$ and $\bigcup_{n=1}^{\infty} F_{n}=X$. Consequently, $\mu\left(F_{n}\right) \uparrow \mu(X)$ when $n \rightarrow \infty$. Since $\mu(X)>0$, there exists an $n \in \mathbb{N}$ such that $\mu\left(F_{n}\right)>0$. But for every $x \in X, f(x) \geq \frac{1}{n} \mathbf{1}_{F_{n}}(x)$. Therefore,

$$
\int_{X} f d \mu \geq \frac{1}{n} \int_{X} \mathbf{1}_{F_{n}} d \mu=\frac{1}{n} \mu\left(F_{n}\right) .
$$

Thus, $\int_{X} f d \mu>0$, as claimed.

Proposition B.4 The set $D_{i}$ is closed with respect to the weak metric in $\Delta\left(A_{i}\right)$ and $P D_{i}$ is closed in $A_{i}$. Moreover, $D_{i}=\Delta\left(P D_{i}\right)$.

Proof. Assume that $D_{i}$ is non-empty, otherwise the statements are obvious. Take a $\rho_{i} \in D_{i}$. For every $\sigma_{-i} \in \Delta\left(A_{-i}\right)$ define

$$
F_{\sigma_{-i}}=\left\{\sigma_{i} \in \Delta\left(A_{i}\right) \mid u_{i}\left(\sigma_{-i}, \sigma_{i}\right)=u_{i}\left(\sigma_{-i}, \rho_{i}\right)\right\} .
$$

Since $u_{i}$ is a continuous function on $\Delta\left(A_{i}\right)$ with respect to the weak metric, $F_{\sigma_{-i}}$ is a closed set. Moreover, $D_{i}=\bigcap_{\sigma_{-i} \in \Delta\left(A_{-i}\right)} F_{\sigma_{-i}}$. Therefore, $D_{i}$ is also a closed set with respect to the weak metric. With a similar argument we can conclude that $P D_{i}$ is a closed subset of $A_{i}$.

Now we show that $D_{i}=\Delta\left(P D_{i}\right)$. Since $\Delta\left(P D_{i}\right) \subseteq D_{i}$, we only need to prove that $\rho_{i} \in D_{i}$ implies $\rho_{i} \in \Delta\left(P D_{i}\right)$, or equivalently $\rho_{i}\left(P D_{i}\right)=1$. Suppose by way of contradiction that $\rho_{i}\left(P D_{i}\right)<1$, implying $\rho_{i}\left(A_{i} \backslash P D_{i}\right)>0$. Take a completely mixed strategy profile $\tau \in \Delta(A)$. Let $c=u_{i}\left(\tau \mid \rho_{i}\right)$. By Lemma B.2 we have

$$
u_{i}\left(\tau \mid x_{i}\right)<u_{i}\left(\tau \mid \rho_{i}\right)=c
$$


for every $x_{i} \in A_{i} \backslash P D_{i}$, while $u_{i}\left(\tau \mid x_{i}\right)=u_{i}\left(\tau \mid \rho_{i}\right)=c$ for every $x_{i} \in P D_{i}$. Then, using Lemma B.3 to get the strict inequality, we have

$$
\begin{aligned}
c=u_{i}\left(\tau \mid \rho_{i}\right) & =\int_{A_{i}} u_{i}\left(\tau \mid x_{i}\right) d \rho_{i} \\
& =\int_{P D_{i}} u_{i}\left(\tau \mid x_{i}\right) d \rho_{i}+\int_{A_{i} \backslash P D_{i}} u_{i}\left(\tau \mid x_{i}\right) d \rho_{i} \\
& <c \rho_{i}\left(P D_{i}\right)+c \rho_{i}\left(A_{i} \backslash P D_{i}\right)=c
\end{aligned}
$$

which is a contradiction.

\section{References}

[1] Aliprantis CD, and KC Border (2006) Infinite dimensional analysis, a hitchhiker's guide. Springer-Verlag Publishers.

[2] Andersson O, C Argenton, and JW Weibull (2010) Robustness to strategic uncertainty. Center DP 2010-70.

[3] Billingsley P (1968) Convergence of probability measures. Wiley Series in Probability and Mathematical Statistics, John Wiley \& Sons.

[4] Carbonell-Nicolau O (2010) Essential equilibria in normal-form games. Journal of Economic Theory 145, 421-431.

[5] Carlsson H, and E van Damme (1993) Global games and equilibrium selection. Econometrica 61, 989-1018.

[6] Compte O, and P Jehiel (2007) Auctions and information acquisition: sealed bid or dynamic formats? The Rand Journal of Economics 38, 355372 .

[7] Crémer J, Y Spiegel, and CZ Zheng (2009) Auctions with costly information acquisition. Economic Theory 38, 41-72.

[8] van Damme E (1996) Stability and perfection of Nash equilibria. SpringerVerlag Publishers.

[9] Govindan S, and R Wilson (2008) Metastable equilibria. Mathematics of Operations Research 33, 787-820. 
[10] Hillas J (1990) On the definition of the strategic stability of equilibria. Econometrica 58, 1365-1391.

[11] Jackson MO, LK Simon, JM Swinkels, and WR Zame (2002) Communication and equilibrium in discontinuous games of incomplete information. Econometrica 70, 1711-1740.

[12] Jiang Jia-He (1962) Essential fixed points of the multivalued mappings. Scientia Sinica 11, 293-298

[13] Kohlberg E, and JF Mertens (1986) On the strategic stability of equilibria. Econometrica 54, 1003-1037

[14] Kreps D, and R Wilson (1982) Sequential equilibria. Econometrica 50, 863894.

[15] Méndez-Naya L, I García-Jurado, and JC Cesco (1995) Perfection of Nash equilibria in continuous games. Mathematical Social Sciences 29, 225-237.

[16] Mertens JF (1989) Stable equilibria - a reformulation. Part I: Definitions and basic properties. Mathematics of Operations Research 14, 575-625.

[17] Mertens JF (1991) Stable equilibria - a reformulation. Part II: Discussion of the definition and futher results. Mathematics of Operations Research $16,694-753$.

[18] Mertens JF (2003) Ordinality in non cooperative games. International Journal of Game Theory 32, 387-430.

[19] Morris S, and HS Shin (2003) Global games: theory and applications. Advances in economics and econometrics, M Dewatripont, L Hansen, and $\mathrm{S}$ Turnovsky (eds), Cambridge University Press, NY.

[20] Myerson RB (1978) Refinements of the Nash equilibrium concept. International Journal of Game Theory 7, 73-80.

[21] Nash JF (1950) Equilibrium points in n-person games. Proceedings from the National Academy of Science USA 36, 48-49.

[22] Nash JF (1951) Noncooperative games. Annals of Mathematics 54:286-295. 
[23] Selten R (1975) Reexamination of the perfectness concept for equilibrium points in extensive games. International Journal of Game Theory 4, 25-55.

[24] Simon LK, and MB Stinchcombe (1995) Equilibrium refinement for infinite normal-form games. Econometrica 63:1421-1443.

[25] Vermeulen AJ, and MJM Jansen (1997) On the invariance of solutions of finite games. Mathematical Social Sciences 33, 251-267.

[26] Wu Wen-Tsün and Jiang Jia-He (1962) Essential equilibrium points of nperson non-cooperative games. Scientia Sinica 11, 1307-1322 